\title{
CHROMOSOME NUMBERS IN BROMELIACEAE ${ }^{1}$
}

\author{
Gregory K. Brown and Amy Jean Gilmartin ${ }^{2,3}$
}

Department of Botany and Rocky Mountain Herbarium, University of Wyoming, Laramie, Wyoming 82071; and ${ }^{2}$ Department of Botany and Marion Ownbey Herbarium, Washington State University, Pullman, Washington 99164

\begin{abstract}
A B S T R A C T
Eighty-three chromosome counts are reported for 72 taxa of the Bromeliaceae. Fifty-eight of these counts are the first known chromosome number reports for their respective taxa. A model of chromosomal evolution in the Bromeliaceae $(n=25)$ is presented. The model is parsimonious and consistent with existing data on meiotic chromosome numbers within the family and in the closely related Velloziaceae $(n=9)$. Two hypothesized paleodiploids $(n=8$ and $n=9)$ hybridized to form a tetraploid that in turn hybridized with the $n=8$ lineage. The resultant $n$ $=25$ is the extant base number for the family. Two alternative hypotheses could explain the unique extant base number $(n=17)$ for Cryptanthus: 1) Cryptanthus represents the paleotetraploid level, i.e., prior to the second round of hybridization, or 2) the lower number represents the result of a more recent series of aneuploid reductions from $n=25$. Given the existence of intergeneric hybrids involving Cryptanthus, aneuploid reduction is the more likely interpretation.
\end{abstract}

RECENT RESEARCH concerning Bromeliaceae systematics and evolution (e.g., Brown and Gilmartin, 1984, 1986; Gilmartin and Brown, $1985,1986 \mathrm{~b}$ ) has sparked renewed interest in the study of Bromeliaceae chromosomes and chromosome evolution. Past chromosome number surveys in the family (i.e., Lindschau, 1933; Gauthé, 1965; Weiss, 1965; Sharma and Ghosh, 1971; Till, 1984) have relied mostly on mitotic material. The only major exception to this was Marchant (1967) who utilized meiotically active microsporocytes. There is great variability in reported mitotic chromosome numbers (Brown and Gilmartin, 1986), and lack of concordance between mitotic and meiotic numbers for some taxa within the family. This variability in mitotic number is reflected in the variable interpretations of chromosome base numbers for the family. Brown and Gilmartin (1986) summarized the previous controversy over base number determination for Bromeliaceae, and discussed the current level of knowledge concerning poly-

\footnotetext{
${ }^{1}$ Received for publication 13 October 1987; revision accepted 28 October 1988.

We thank a dedicated group of field collaborators, without whom this project would not have been possible: James Ackerman, Puerto Rico; Stephan Beck, Bolivia; Olga Benavides, Colombia; Elizabeth Bravo, Ecuador; David Brunner, Paraguay; I. Chacon, Costa Rica; Hermes Cuadros, Colombia; Linda Escobar, Colombia; Gert Hatschbach, Brazil; Stephen Koch, Mexico; Gustavo Martinelli, Brazil; Fernando Ortiz, Ecuador; Isidoro Sanchez Vega, Peru; and Rosa Subils, Argentina. Expert technical help was supplied by Carol Annable. We thank Ron Hartman, Don Hauber, and two anonymous reviewers for their comments. This work was supported by collaborative research grants BSR-8607187 (GKB) and BSR-8407573 (AJG) from the National Science Foundation.

${ }^{3}$ Deceased 10 February 1989.
}

ploidy, chromosome size bimodality, and the correlation of nonconcordance in meiotic and mitotic chromosome numbers with the epiphytic mode of growth.

The purpose of this paper is to describe results of an ongoing meiotic chromosome number survey within the Bromeliaceae, and especially subfamily Tillandsioideae. We also present a model for chromosome base number evolution for the family that is consistent and parsimonious with existing data.

METHODS AND MATERIALS - Floral buds were collected in the field, or obtained from cultivated material at Marie Selby Botanical Gardens, Sarasota, Florida (SEL). Buds were fixed in field-mixed Farmer's solution $(100 \% \mathrm{EtOH}$ : glacial acetic acid; 3:1/v:v) to which a drop of saturated aqueous ferric chloride $\left(\mathrm{FeCl}_{3}-6 \mathrm{H}_{2} \mathrm{O}\right)$ had been added. The latter enhances chromosome stainability. After a minimum of 24 $\mathrm{hr}$, fixed buds are transferred to $70 \% \mathrm{EtOH}$. See Gilmartin and Brown (1986a) for a complete description of the field collaborator network and its operation.

For chromosome squash preparations, individual anthers were removed from the bud in $70 \% \mathrm{EtOH}$ and transferred to a pool of $1 \%$ acetic carmine on a microscope slide. While in the stain, the anther is cut transversely in half. Using ultrafine-tipped needle and forceps, the sporogenous masses are squeezed from each microsporangium through the median transverse cut. The sporogenous masses are positioned toward the center of the stain pool and a coverslip and gentle finger pressure are applied. The preparation is further flattened by passing the slide through an alcohol flame sev- 
eral times. Heating the slide helps to rupture the callose that encapsulates the microsporocyte. Squashes were examined with phase contrast microscopy, and documented using Kodak Technical Pan 2415 and drawings. As standard practice, a minimum of five microsporocytes with unambiguous meiotic figures (usually in diplotene, diakinesis, metaphase I, or metaphase II) serve as the basis for chromosome number determination. In cases where a new chromosome number (e.g., Tillandsia leiboldiana) or abnormality (e.g., B-chromosomes or fragments) was encountered, as many as 18 unambiguous meiotic figures were documented. Voucher herbarium specimens are at WS unless otherwise indicated (see Table 1). All graphic documentation of chromosomes is at RM. The nomenclature followed here is that of Smith and Downs $(1974,1977,1979)$.

RESULTS-Eighty-three chromosome counts are reported for 72 taxa (Table 1). For the most part, reports are either the first known published chromosome number, or represent a previously unreported number for a taxon. Representative squash preparations are shown in Fig. 1-4.

Bromelioideae-Approximately $15 \%$ of the ca. 570 species within Bromelioideae are known by at least one chromosome number report. Unfortunately, these reports are not evenly spread across the 27 genera recognized by Smith and Downs (1979). Most come from five genera (Aechmea, Billbergia, Cryptanthus, Neoregelia, Nidularium), while $14(51 \%)$ of the genera are unknown chromosomally (Andrea, 1 sp.; Androlepis, 1 sp.; Araeococcus, 5 spp.; Disteganthus, 2 spp.; Fascicularia, 5 spp.; Fernseea, 1 sp.; Greigia, 26 spp.; Hohenbergia, 40 spp.; Hohenbergiopsis, 1 sp.; Neoglaziovia, 2 spp.; Ochagavia, 3 spp.; Orthophytum, 17 spp.; Ronnbergia, 8 spp.; Wittrockia, 7 spp.).

Except for Cryptanthus and Aechmea tillandsioides (Martius ex Schultes f.) Baker, all Bromelioideae genera thus far studied appear to have a meiotically established extant base number of $x=25$ (see Brown and Gilmartin, 1986). Cryptanthus is anomalous in having a base of $x=17$ (Marchant, 1967) and the possible significance of this is discussed later. Aechmea tillandsioides ( $n=21$; Marchant, 1967) would appear to be an aneuploid derivative.

Pitcairnioideae-Chromosome counts are available for six pitcairnioid genera (Deuterocohnia, Dyckia, Fosterella, Hechtia, Pitcairnia, Puya). Members of the subfamily are thus far homogeneous for the base number of $x=$ 25 (also see Brown and Gilmartin, 1986). All repeat chromosome number reports made here for Pitcairnioideae taxa corroborate one or more previous counts (i.e., Lindschau, 1933; Di Fulvio, 1967; Marchant, 1967; Brown et al., 1984). With the addition of new genera Brewcaria and Steyerbromelia published since Smith and Downs (1974), Pitcairnioideae contains 15 genera. Chromosome data are lacking for the following genera: Abromeitiella (2 spp.), Ayensua (1 sp.), Brewcaria (1 sp.), Brocchinia (18 spp.), Connellia (4 spp.), Cottendorfia (24 spp.), Encholirium (12 spp.), Navia (74 spp.), and Steyerbromelia (1 sp.).

Tillandsioideae-With over 800 species in six genera (Catopsis, 19 spp.; Glomeropitcairnia, 2 spp.; Guzmania, 126 spp.; Mezobromelia, 3 spp.; Tillandsia, 410 spp.; Vriesea, 250 spp.), this is the largest of the subfamilies. Published chromosome number information now is available for all but Mezobromelia. The primary focus of this research is Tillandsia, the largest genus in the family.

A comparison of published chromosome number data for Tillandsia (Brown and Gilmartin, 1986) has revealed a striking discrepancy between mitotic (root tip) and meiotic chromosome numbers. Prior attempts to discover trends of chromosomal evolution within Tillandsioideae (e.g., Lindschau, 1933; Gauthé, 1965) had been hindered by this variability in mitotic chromosome number reports (see Brown and Gilmartin, 1986, for additional discussion). An explicit goal of our research has been to determine the level of meiotic chromosome number variability within Tillandsia.

The count of $n=22$ for T. complanata (subg. Allardtia) differs from an earlier report (Brown et al., 1984; $n=20$ ). Tillandsia complanata is a wide ranging forest epiphyte (Greater Antilles, Costa Rica to Bolivia and N. Brazil; Smith and Downs, 1977) and may comprise cytogeographic races. These two counts correspond closely to the known northern and southern limits for this species.

The report for $T$. leiboldiana (Fig. 2) is the first record of $n=19$ within the family. This, and the $T$. complanata chromosome numbers presumably arose via dysploidy from an ancestral $n=25$. We view such chromosome numbers as being derived. The two reports for $T$. complanata and the remaining 13 reports for species of the subg. Allardtia presented here are the only known chromosome number reports for this subgenus.

The chromosome number reports for $T$. lorentziana and $T$. vernicosa $(n=25)$ are the 
TABLE 1. Chromosome number reports: Bromeliaceae

\begin{tabular}{|c|c|c|}
\hline Taxon & $n$ & Voucher \\
\hline \multicolumn{3}{|l|}{ Bromelioideae } \\
\hline $\begin{array}{l}{ }^{\mathrm{a}} \text { Aechmea purpureo-rosea (Hook.) } \\
\text { Wawra }\end{array}$ & 25 & $\begin{array}{l}\text { Brazil: Copuva Bahia } \\
\text { Hatschbach } 48762\end{array}$ \\
\hline $\begin{array}{l}\text { Ananas ananassoides (Baker) Lyman } \\
\text { B. Smith }\end{array}$ & & $\begin{array}{l}\text { Paraguay: Dpto. Amambay } \\
\text { Brunner } 1488\end{array}$ \\
\hline var. ananassoides & 25 & \\
\hline \multicolumn{3}{|l|}{ Pitcairnioideae } \\
\hline a Deuterocohnia haumanii Castell. & 25 & $\begin{array}{l}\text { Argentina: Prov. Salta } \\
\text { Subils } 3556\end{array}$ \\
\hline D. longipetala (Baker) $\mathrm{Mez}$ & 25 & $\begin{array}{l}\text { Argentina: Prov. Cordoba } \\
\text { Di Fulvio \& Subils } 748 \& 756\end{array}$ \\
\hline $\begin{array}{l}\text { a Dyckia microcalyx Baker } \\
\text { var. microcalyx }\end{array}$ & 25 & $\begin{array}{l}\text { Paraguay: Dpto. Paraguari } \\
\text { Brunner } 1263\end{array}$ \\
\hline D. tomentella $\mathrm{Mez}$ & 25 & $\begin{array}{l}\text { Paraguay: Dpto. Presidente Hayes } \\
\text { Brunner } 1346\end{array}$ \\
\hline${ }^{\mathrm{a}}$ D. velascana $\mathrm{Mez}$ & 25 & $\begin{array}{l}\text { Argentina: Prov. Salta } \\
\text { Subils } 3573\end{array}$ \\
\hline${ }^{\mathrm{a}}$ Fosterella rusbyi (Mez) & 25 & Bolivia: Dpto. La Paz \\
\hline Lyman B. Smith & & Beck 12076 \\
\hline aPitcairnia bakeri (André) & 25 & Ecuador: Prov. Pichincha \\
\hline André ex Mez & & Bravo 843 \\
\hline${ }^{a} P$. dendroidea André & 25 & $\begin{array}{l}\text { Colombia: Dpto. Cauca } \\
\text { Escobar et al. } 4338\end{array}$ \\
\hline P. flammea Lindley & & Brazil: Edo. Rio de Janeiro \\
\hline var. roezlii (E. Morren) Lyman B. Smith & 25 & Martinelli 11567 \\
\hline${ }^{\text {a } P}$. poortmanii André & 25 & $\begin{array}{l}\text { Ecuador: Prov. Zamora-Chinchipe } \\
\text { Peters } 200\end{array}$ \\
\hline${ }^{\text {a}} P$. sceptrigera $\mathrm{Mez}$ & 25 & $\begin{array}{l}\text { Ecuador: Prov. Pichincha } \\
\text { Ortiz } 837\end{array}$ \\
\hline a Puya eryngioides André & 25 & $\begin{array}{l}\text { Ecuador: Prov. Loja } \\
\text { Peters } 002\end{array}$ \\
\hline 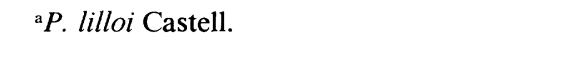 & 25 & $\begin{array}{l}\text { Argentina: Prov. Salta } \\
\quad \text { Subils } 3596\end{array}$ \\
\hline P. spathacea (Griseb.) Mez & 25 & $\begin{array}{l}\text { Argentina: Prov. Cordoba } \\
\text { Di Fulvio \& Subils } 751\end{array}$ \\
\hline \multicolumn{3}{|l|}{ Tillandsioideae } \\
\hline $\begin{array}{c}\text { aCatopsis sessiliflora (Ruíz } \\
\text { Lopez \& Pavón) Mez }\end{array}$ & 25 & $\begin{array}{l}\text { Mexico: Edo. Puebla } \\
\text { Koch \& Wendt } 8513\end{array}$ \\
\hline${ }^{\mathrm{a}} C$. nitida (Hook.) Griseb. & 25 & $\begin{array}{l}\text { Mexico: Edo. Oaxaca } \\
\text { Koch } 86124\end{array}$ \\
\hline${ }^{\mathrm{a}}$ Guzmania mitis Lyman B. Smith & 25 & $\begin{array}{l}\text { Colombia: Dpto. Magdalena } \\
\text { Cuadros } 3165\end{array}$ \\
\hline $\begin{array}{l}{ }^{\mathrm{a}} G \text {. multiflora (André) } \\
\text { André ex } \mathrm{Mez} \\
\text { Tillandsia } \text { subg. Allardtia }\end{array}$ & 25 & $\begin{array}{l}\text { Colombia: Dpto. Antioquia } \\
\text { Escobar et al. } 3944\end{array}$ \\
\hline${ }^{\mathrm{a}} T$. adpressiflora $\mathrm{Mez}$ & 25 & $\begin{array}{l}\text { Ecuador: Prov. Zamora-Chinchipe } \\
\text { Peters } 1300\end{array}$ \\
\hline $\begin{array}{l}{ }^{\mathrm{b}} T \text {. complanata Benth. } \\
\text { subsp. complanata }\end{array}$ & 22 & $\begin{array}{l}\text { Costa Rica: Cartago Alto Coris } \\
\text { Chacon } 1754\end{array}$ \\
\hline${ }^{\mathrm{a}} T$. deppeana Steudel & 25 & $\begin{array}{l}\text { Mexico: Edo. Puebla } \\
\text { Koch \& Wendt } 859\end{array}$ \\
\hline${ }^{\mathrm{a}} T$. dudleyi Lyman B. Smith & 25 & $\begin{array}{l}\text { Peru: Dpto. Cajamarca } \\
\text { Sanchez Vega } 4141\end{array}$ \\
\hline${ }^{\mathrm{a}} T$. fendleri Griseb. & 25 & $\begin{array}{l}\text { Peru: Dpto. Cajamarca } \\
\text { Sanchez Vega } 3660\end{array}$ \\
\hline${ }^{\mathrm{a}} T$. incarnata Kunth & 25 & $\begin{array}{l}\text { Ecuador: Prov. Pichincha } \\
\text { Bravo } 817\end{array}$ \\
\hline \multicolumn{3}{|l|}{ T. latifolia Meyen } \\
\hline${ }^{a}$ var. divaricata (Benth.) $\mathrm{Mez}$ & 25 & $\begin{array}{l}\text { Peru: Dpto. Cajamarca } \\
\text { Sanchez Vega } 3815\end{array}$ \\
\hline var. latifolia & 25 & $\begin{array}{l}\text { Peru: Dpto. La Libertad } \\
\text { Sanchez Vega } 3863\end{array}$ \\
\hline${ }^{\mathrm{a}} T$. leiboldiana Schldl. & & Mexico: Edo. Puebla \\
\hline var. leiboldiana & 19 & Koch \& Wendt 8514 \\
\hline
\end{tabular}


TABle 1. Continued

\begin{tabular}{|c|c|c|}
\hline Taxon & $n$ & Voucher \\
\hline${ }^{\mathrm{a}} T$. plumosa Baker & 25 & $\begin{array}{l}\text { Mexico: Edo. Oaxaca } \\
\text { Peterson \& Annable } 4671\end{array}$ \\
\hline${ }^{\mathrm{a}} T$. pyramidata André & 25 & $\begin{array}{l}\text { Ecuador: Prov. Pichincha } \\
\text { Ortiz } 834\end{array}$ \\
\hline${ }^{\mathrm{a}} T$. rubella Baker & 25 & $\begin{array}{l}\text { Colombia: Dpto. Magdalena } \\
\text { Cuadros } 2020\end{array}$ \\
\hline${ }^{\mathrm{a}} T$. sigmoidea Lyman B. Smith & 25 & $\begin{array}{l}\text { Colombia: Dpto. Magdalena } \\
\text { Cuadros } 3160\end{array}$ \\
\hline${ }^{\mathrm{a}} T$. stenoura Harms & & Colombia: Dpto. Magdalena \\
\hline var. stenoura & 25 & Cuadros 2016 \\
\hline \multirow[t]{2}{*}{${ }^{\mathrm{a}} T$. tovarensis $\mathrm{Mez}$} & 25 & $\begin{array}{l}\text { Peru: Dpto. Cajamarca } \\
\text { Sanchez Vega } 3679\end{array}$ \\
\hline & 25 & $\begin{array}{l}\text { Colombia: Dpto. Magdalena } \\
\text { Cuadros } 3166\end{array}$ \\
\hline \multicolumn{3}{|l|}{ Subg. Anoplophytum } \\
\hline${ }^{\mathrm{a}} T$. lorentziana Griseb. & 25 & $\begin{array}{l}\text { Argentina: Prov. Catamarca } \\
\text { Subils } 3661\end{array}$ \\
\hline${ }^{\mathrm{a}} T$. vernicosa Baker & 25 & $\begin{array}{l}\text { Bolivia: Dpto. La Paz } \\
\text { Beck } 12072\end{array}$ \\
\hline \multicolumn{3}{|l|}{ Subg. Diaphoranthema } \\
\hline $\begin{array}{l}{ }^{a} T \text {. capillaris Ruiz Lopez \& Pavón } \\
\text { forma hieronymii }(\mathrm{Mez}) \text { Lyman B. Smith }\end{array}$ & 50 & $\begin{array}{l}\text { Argentina: Prov. Cordoba } \\
\text { Di Fulvio \& Subils } 770\end{array}$ \\
\hline $T$. recurvata $(\mathrm{L}.) \mathrm{L}$. & 25 & $\begin{array}{l}\text { Ecuador: Prov. Pichincha } \\
\text { Bravo } 816\end{array}$ \\
\hline \multicolumn{3}{|l|}{${ }^{\mathrm{a}} T$. tricholepis Baker } \\
\hline \multirow[t]{2}{*}{ var. tricholepis } & 50 & $\begin{array}{l}\text { Argentina: Prov. Catamarca } \\
\text { Subils } 3682\end{array}$ \\
\hline & 50 & $\begin{array}{l}\text { Argentina: Prov. Salta } \\
\text { Subils } 3572\end{array}$ \\
\hline Subg. Phytarrhiza & & \\
\hline${ }^{\mathrm{a}} T$. dodsonii Lyman B. Smith & 25 & $\begin{array}{l}\text { Colombia: Dpto. Nario } \\
\text { Benavides } 4304\end{array}$ \\
\hline \multicolumn{3}{|l|}{${ }^{\mathrm{a}} T$. duratii Vis. } \\
\hline \multirow[t]{2}{*}{ var. duratii } & 25 & $\begin{array}{l}\text { Bolivia: Dpto. La Paz } \\
\text { Beck } 11211\end{array}$ \\
\hline & 25 & $\begin{array}{l}\text { Argentina: Prov. Salta } \\
\quad \text { Palaci } 547\end{array}$ \\
\hline${ }^{\mathrm{a}} T$. humilis C. Presl & 25 & $\begin{array}{l}\text { Peru: Dpto. Cajamarca } \\
\text { Sanchez Vega } 3834\end{array}$ \\
\hline $\begin{array}{l}\text { T. monadelpha (E. Morren) } \\
\text { Baker }\end{array}$ & 25 & $\begin{array}{l}\text { Colombia: Mpio. Tumaco } \\
\text { Benavides } 6700\end{array}$ \\
\hline${ }^{\mathrm{a}} T$. purpurea Ruíz Lopez \& Pavón & 25 & $\begin{array}{l}\text { Peru: Dpto. Cajamarca } \\
\text { Sanchez Vega } 3666,4140\end{array}$ \\
\hline${ }^{\mathrm{a}} T$. reichenbachii Baker & 25 & $\begin{array}{l}\text { Argentina: Prov. Salta } \\
\text { Subils } 3574\end{array}$ \\
\hline T. scaligera Mez \& Sodiro & $\begin{array}{l}25+2-10 \\
\text { fragments }\end{array}$ & $\begin{array}{l}\text { Ecuador: Rio Palenque } \\
\text { Dodson s.n. (SEL 81-822) }\end{array}$ \\
\hline \multicolumn{3}{|l|}{ Subg. Pseudalcantarea } \\
\hline T. viridiflora (Beer) Baker & 25 & $\begin{array}{l}\text { Mexico: Edo. Oaxaca } \\
\text { Koch } 86123\end{array}$ \\
\hline \multicolumn{3}{|l|}{ Subg. Pseudo-Catopsis } \\
\hline${ }^{\mathrm{a}} T$. diffusa Lyman B. Smith & 25 & $\begin{array}{l}\text { Peru: Dpto. Cajamarca } \\
\text { Sanchez Vega } 3814\end{array}$ \\
\hline \multicolumn{3}{|l|}{${ }^{\mathrm{a}} T$. multiflora Benth. } \\
\hline var. tomensis Lyman B. Smith & 25 & $\begin{array}{l}\text { Peru: Dpto. Cajamarca } \\
\text { Sanchez Vega } 3864\end{array}$ \\
\hline${ }^{\mathrm{a}} T$. riocreuxii André & 25 & $\begin{array}{l}\text { Colombia: Mpio. Belco } \\
\text { Escobar } 7200\end{array}$ \\
\hline${ }^{\mathrm{a}} T$. ropalocarpa André & 25 & $\begin{array}{l}\text { Peru: Dpto. Cajamarca } \\
\quad \text { Sanchez Vega } 3865\end{array}$ \\
\hline${ }^{\mathrm{a}} T$. subalata André & 25 & $\begin{array}{l}\text { Ecuador: Prov. Pichincha } \\
\text { Ortiz } 8311\end{array}$ \\
\hline $\begin{array}{l}\text { Subg. Tillandsia } \\
{ }^{\mathrm{a}} T . \text { baileyi Rose ex Small }\end{array}$ & 25 & $\begin{array}{l}\text { U.S.A.: Texas } \\
\text { Gardner } 1165\end{array}$ \\
\hline
\end{tabular}


TABLE 1. Continued

\begin{tabular}{|c|c|c|}
\hline Taxon & $n$ & Voucher \\
\hline${ }^{a} T$. bourgaei Baker & 25 & $\begin{array}{l}\text { Mexico: Edo. Sinaloa } \\
\text { Peterson \& Annable } 4156\end{array}$ \\
\hline${ }^{\mathrm{a}} T$. caput-medusae E. Morren & 25 & $\begin{array}{l}\text { El Salvador: Prov. Santa Ana } \\
\text { Mazariego } 1\end{array}$ \\
\hline \multirow[t]{2}{*}{$\begin{array}{l}\text { T. fasciculata Sw. } \\
\text { var. fasciculata }\end{array}$} & 25 & $\begin{array}{l}\text { Costa Rica: San Jose } \\
\text { Chacon 1751 }\end{array}$ \\
\hline & 25 & $\begin{array}{l}\text { Mexico: Edo. Chiapas } \\
\text { Peterson \& Annable } 4705\end{array}$ \\
\hline${ }^{\mathrm{a}} T$. filifolia Schldl. \& Cham. & 25 & $\begin{array}{l}\text { Mexico: Edo. Puebla } \\
\text { Koch \& Wendt } 854\end{array}$ \\
\hline T. imperialis E. Morren ex Mez & 25 & $\begin{array}{l}\text { Mexico: Edo. Hidalgo } \\
\text { Koch } 842\end{array}$ \\
\hline $\begin{array}{l}\text { T. juncea (Ruíz Lopez \& Pavón) } \\
\text { Poiret }\end{array}$ & 25 & $\begin{array}{l}\text { Mexico: Edo. Chiapas } \\
\text { Martinez } 17622\end{array}$ \\
\hline${ }^{\mathrm{a}} T$. kirchhoffiana Wittm. & 25 & $\begin{array}{l}\text { Mexico: Edo. Mexico } \\
\text { Koch \& Fryxell } 8331\end{array}$ \\
\hline${ }^{\mathrm{a}} T$. mexicana Lyman B. Smith & 25 & $\begin{array}{l}\text { Mexico: Edo. Oaxaca } \\
\quad \text { Peterson \& Annable } 4672\end{array}$ \\
\hline \multirow[t]{2}{*}{${ }^{\mathrm{a}} T$. polystachia (L.) L. } & 25 & $\begin{array}{l}\text { Mexico: Edo. Mexico } \\
\text { Koch \& Fryxell } 8330\end{array}$ \\
\hline & $\begin{array}{c}25+ \\
\text { B chro- } \\
\text { mosomes }\end{array}$ & $\begin{array}{l}\text { Colombia: Dpto. Antioquia } \\
\text { Escobar et al. } 3659\end{array}$ \\
\hline \multirow[t]{2}{*}{${ }^{\mathrm{a}}$ T. utriculata $\mathrm{L}$. } & 25 & $\begin{array}{l}\text { West Indies: Monserrat } \\
\text { Gilmartin } 2990\end{array}$ \\
\hline & 25 & $\begin{array}{l}\text { Puerto Rico: Dorado } \\
\text { Ackerman \& Montalov } 2004\end{array}$ \\
\hline \multirow[t]{3}{*}{${ }^{\mathrm{a}} T$. violacea Baker } & 25 & $\begin{array}{l}\text { Mexico: Edo. Hidalgo } \\
\text { Koch } 8314\end{array}$ \\
\hline & 25 & $\begin{array}{l}\text { Mexico: Edo. Oaxaca } \\
\text { Koch } 86107\end{array}$ \\
\hline & 25 & $\begin{array}{l}\text { Mexico: Edo. Michoacan } \\
\text { Peterson \& Annable } 4628\end{array}$ \\
\hline \multirow[t]{3}{*}{$\begin{array}{l}\text { Vriesea elata (Baker) } \\
\text { Lyman B. Smith }\end{array}$} & 25 & $\begin{array}{l}\text { Colombia: Dpto. Antioquia } \\
\text { Escobar et al. } 3661\end{array}$ \\
\hline & 25 & $\begin{array}{l}\text { Colombia: Dpto. Guatape } \\
\text { Escobar } 7226\end{array}$ \\
\hline & 25 & $\begin{array}{l}\text { Colombia: Dpto. Magdalena } \\
\text { Cuadros } 3167\end{array}$ \\
\hline a $V$. haematina Lyman B. Smith & 25 & $\begin{array}{l}\text { Brazil: Edo. Rio de Janeiro } \\
\text { Martinelli } 10644\end{array}$ \\
\hline $\begin{array}{l}\text { a } V \text {. harmsiana (Lyman B. Smith) } \\
\text { Lyman B. Smith }\end{array}$ & 25 & $\begin{array}{l}\text { Peru: Dpto. Cajamarca } \\
\text { Sanchez Vega } 3656\end{array}$ \\
\hline${ }^{a} V$. neoglutinosa $\mathrm{Mez}$ & 25 & $\begin{array}{l}\text { Brazil: Edo. Rio de Janeiro } \\
\text { Martinelli } 11683\end{array}$ \\
\hline \multicolumn{3}{|l|}{ a $V$. psittacina (Hook.) Lindley } \\
\hline var. psittacina & 25 & $\begin{array}{l}\text { Brazil: Edo. Rio de Janeiro } \\
\text { Martinelli } 11573\end{array}$ \\
\hline${ }^{\mathrm{a}} V$. werkleana $\mathrm{Mez}$ & 25 & $\begin{array}{l}\text { Mexico: Edo. Chiapas } \\
\text { Peterson \& Annable } 4708\end{array}$ \\
\hline $\begin{array}{l}\text { a. zamorensis (Lyman B. Smith) } \\
\text { Lyman B. Smith }\end{array}$ & 25 & $\begin{array}{l}\text { Ecuador: Prov. Zamora-Chinchipe } \\
\text { Peters } 202\end{array}$ \\
\hline
\end{tabular}

a First chromosome count for taxon.

b New number for taxon.

third and fourth counts for subgenus Anoplophytum. The earlier reports are for T. aeranthos ( $2 n=64$; Gauthé, 1965, reported as T. dianthoidea Rossi) and T. tenuifolia L. $(n=25+$ 1-2 fragments; Marchant, 1967).

For the first time, tetraploidy in Tillandsia is documented from meiotic material in T. cap- illaris (subg. Diaphoranthema). Till (1984) reported mitotic "tetraploid level" numbers $(2 n$ = 84-96) for this same species.

The reports for $T$. recurvata and $T$. tricholepis are the first meiotic counts for these species. They corroborate the ploidy levels determined by Till (1984). An apparent polyploid 


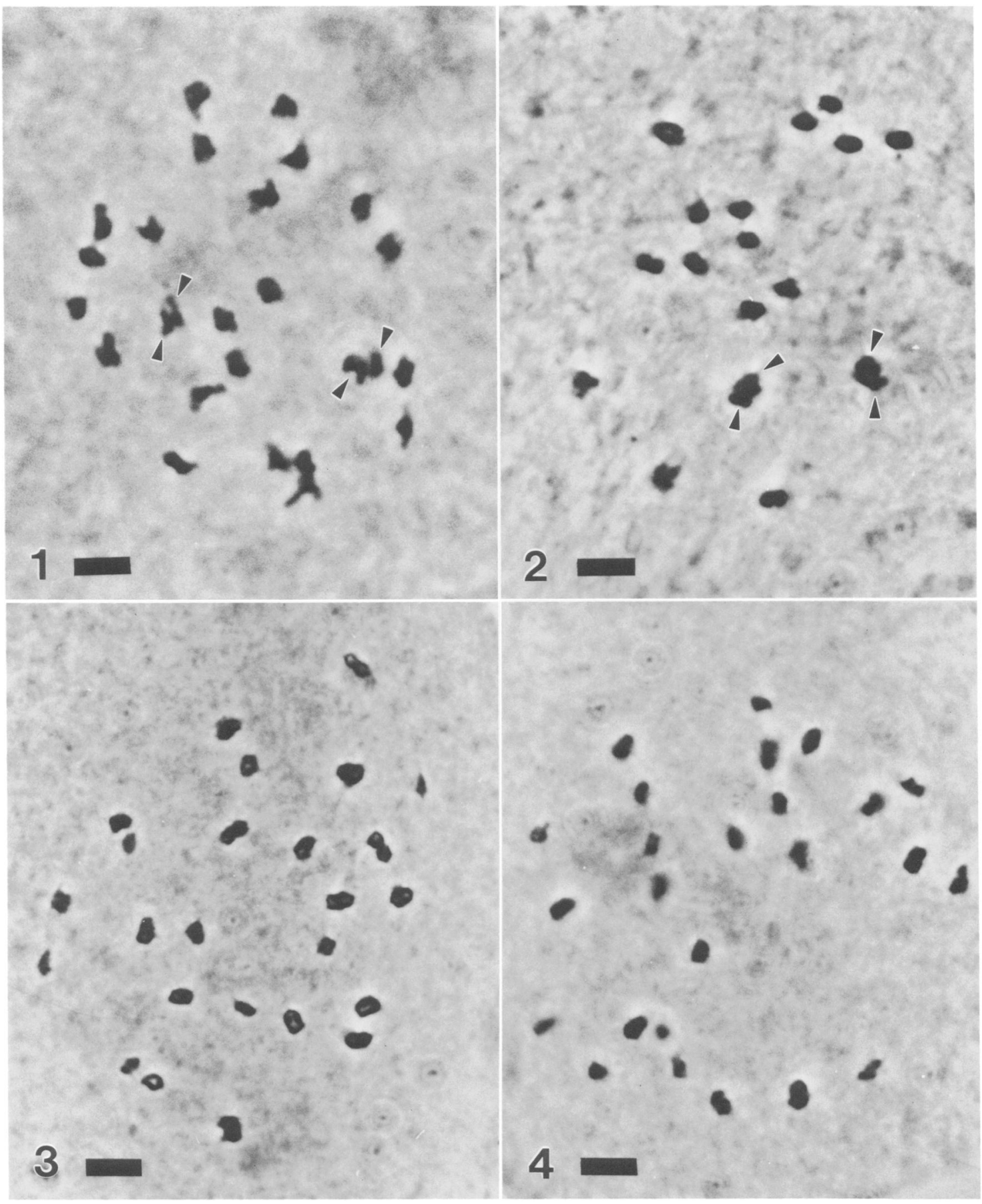

Fig. 1-4. Representative meiotic chromosome squash preparations from Bromeliaceae. Bar scale equal $5 \mu \mathrm{m} .1$. Puya eryngioides, diakinesis, $2 n=25_{\mathrm{II}}$. 2. Tilliandia leiboldiana var. leiboldiana, diakinesis, $2 n=19_{\mathrm{II}}$. 3. Tillandsia incarnata, diakinesis, $2 n=25_{\mathrm{II}}$. 4. Tillandsia rubella, diakinesis, $2 n=25_{\mathrm{II}}$. Arrows indicate separate, overlapping bivalents. Vouchers are listed in Table 1.

series exists in T. tricholepis where Till (1984) reported $2 n=50$ (diploid) for variety macrophylla and $2 n=90$ and 94 ("tetraploid-level") for variety tricholepis.
The count of $n=25+2-10$ fragments for $T$. scaligera is the second for this species. This report notes the variable number of chromosome fragments present at metaphase I. Our 
initial report for $T$. scaligera (Brown et al., 1984) was published as $n=$ c. 25 . That report was submitted in manuscript as " $n=25+6-$ 11 fragments at metaphase I," but editorial policy dictated omission of this information. It should be noted that the two $T$. scaligera collections came from different sites in Ecuador, collected during different years, and both are maintained in cultivation at SEL. Study of additional $T$. scaligera populations is necessary before attempting to interpret the significance of these fragments.

Tillandsia subgenera Pseudalcantarea (4 spp.) and Pseudo-Catopsis (48 spp.) are poorly understood. The chromosome counts presented here are the first for these two subgenera.

Within subg. Tillandsia, the meiotic number report for $T$. fasciculata $(n=25)$ differs from two earlier reports. Gauthé (1965) reported $2 n$ $=64$ for $T$. fasciculata without varietal designation, and $2 n=56$ for variety venosispica (reported as $T$. compressa Bert. ex Schultes). Tillandsia fasciculata is a polymorphic species that includes at least ten varieties (Smith and Downs, 1977). Its geographical range includes Mexico, Central America, Florida, Caribbean Islands, and northern South America. All varieties are epiphytic, with two (laxispica and venosispica) that are sometimes saxicolous.

A similar lack of concordance between meiotic and mitotic chromosome numbers is encountered in T. imperialis. Gauthe (1965) reported a mitotic $2 n=64$, while we report a gametic number of $n=25$. Tillandsia imperialis typically is epiphytic in forests and may be saxicolous (Smith and Downs, 1977).

The first meiotic and diploid level count for $T$. juncea is reported here. Both Lindschau (1933) and Gauthé (1965) reported (as T. juncifolia) $2 n=96$ for this species.

The first two chromosome number reports for $T$. polystachia merit mention because of B-chromosomes found in the Colombian collections (Escobar et al., 3659). The number of B-chromosomes varied between floral buds from an observed low number of two, to as many as six per cell. There were also indications that the B-chromosome number varied between anthers in the same bud. The collection from Mexico (Koch and Fryxell 8330) showed 25 bivalents without B-chromosomes.

Discussion - The chromosome numbers presented here continue to support Marchant's (1967) proposal that $x=25$ is the base number for the family. We view this as the extant base number, since, according to criteria put forth by Grant $(1963,1981), x=25$ is considered to be polyploid in origin.

McWilliams (1974) presented a scheme for bromeliad chromosome number evolution that attempted to account for all numbers then available. The scheme, hypothetically ancestral at $x=8$, is necessarily complicated by attempts to account for reported mitotic numbers (i.e., $2 n=32,48,56,64$ ) which do not agree with the growing data for meiotic numbers. In this regard, the report by Billings (1904) of $n=16$ for Tillandsia usneoides, the first published chromosome number for the family and one which has not yet been corroborated, appears to have lent an unfortunate bias to some subsequent chromosomal interpretations. The Billings (1904) report would suggest a base number of 8 for the lineage. Curiously, a series of later mitotic studies (e.g., Lindschau, 1933; Gauthé, 1965; Weiss, 1965) report an abundance of numbers at the hexaploid, septaploid and octaploid levels based on $x=8$ (i.e., $2 n=48,56,64$ ). We should note that chromosomal races may be present within this morphologically variable and most widespread species in the family (Smith, 1934). Billings studied material collected in Louisiana, while plants for the $T$. usneoides count made by Till $(2 n=50 ; 1984)$ came from Argentina or Bolivia.

For the interpretation of chromosome number evolution, the use of somatically determined (holdfast-root tips) chromosome number reports (e.g., Lindschau, 1933; Gauthé, 1965; Weiss, 1965; Sharma and Ghosh, 1971) that do not agree with meiotically determined numbers would seem to be an unwise practice. Whether apparent meiotic and mitotic chromosome number nonconcordance in epiphytic bromeliads is real (e.g., unstable B-chromosomes), an artifact, or is the resultant combination of bias and/or inaccurate observation by some previous workers has not yet been clearly established. Research is now in progress to examine this problem. At present, we feel that the most prudent approach is to base our interpretations and hypotheses about chromosome evolution for the Bromeliaceae on meiotic chromosome numbers (e.g., Marchant, 1967; Brown and Gilmartin, 1983; Brown et al., 1984; Varadarajan and Brown, 1985; the reports here).

Figure 5 outlines a hypothesis to account for the extant Bromeliaceae chromosome number, $x=25$. The model is dibasic and involves hybridization and polyploidy of paleodiploid base numbers $x=8$ and 9 to yield a paleotetraploid $(n=17)$. This was followed by hybridization between a paleodiploid $(n=8)$ and the paleotetraploid lineage $(n=17)$ with polyploid stabilization at the hexaploid level $(n=$ 25). The most recent common ancestor for the three subfamilies was hexaploid. Electropho- 


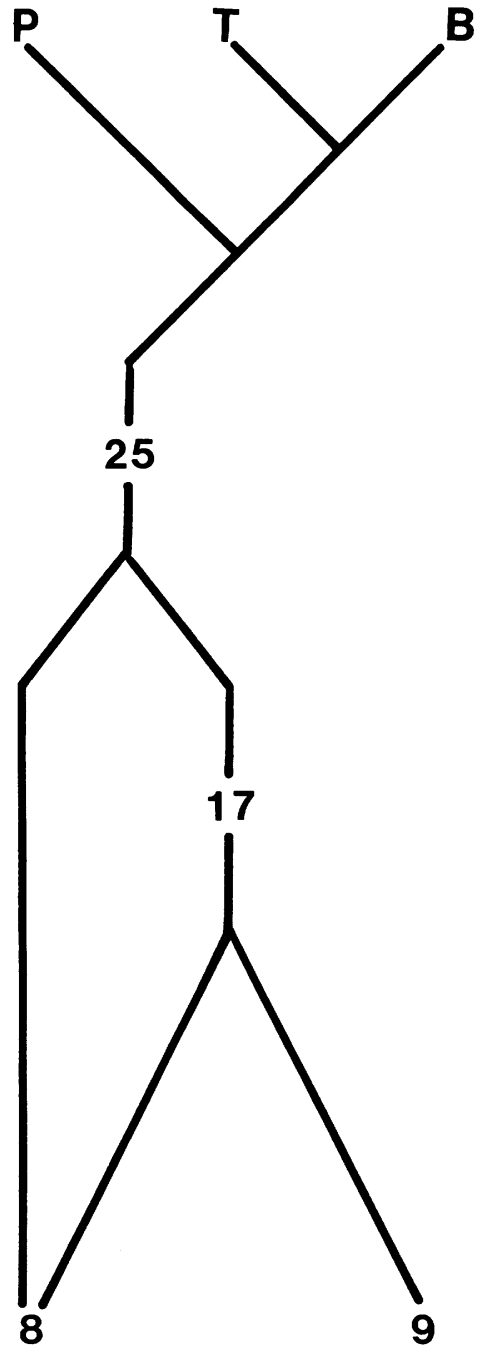

Fig. 5. Proposed model for chromosome base number evolution in Bromeliales. The extant base number $n=25$ is synapomorphic for Bromeliaceae and hypothetically derived from hybridization and polyploidy involving a paleodiploid $(n=8)$ and paleotetraploid $(n=17)$. The dibasic paleotetraploid developed from hypothesized hybridization and polyploidy involving paleodiploids $n=8$ and $n$ $=9$. P, Pitcairnioideae; T, Tillandsioideae; $\mathrm{B}$, Bromelioideae.

retic studies of two Tillandsia species by Soltis et al. (1987) have shown these to be isozymically diploid except for an additional isozyme for PGM in $T$. recurvata. This electrophoretic data suggests that "diploidization" of the dibasic paleohexaploid has occurred.

Other hypotheses are possible, but this one is consistent with the data and existing notions (e.g., Goldblatt and Poston, 1988) concerning base numbers (i.e., $x=8$ and/or 9) and chromosomal evolution within the superorder Bromeliiflorae of Dahlgren, Clifford, and Yeo
(1985). Goldblatt and Poston (1988) have proposed a hypothesis for chromosome number evolution in the Velloziaceae that recognizes $x=9$ as the paleodiploid base. Aware of an increasingly apparent close (possibly sister taxon) relationship between Bromeliaceae and Velloziaceae (Dahlgren et al., 1985; Gilmartin and Brown, 1987; Menezes, personal communication; Frolick and Barthlott, 1987; Ranker et al., 1988), we speculate that the paleodiploid $n=9$ genome is shared by these two families. This notion is supported by restriction fragment analyses of chloroplast DNA (Ranker et al., 1988).

Chromosome numbers at the hypothesized paleodiploid level (i.e., $n=8$ or 9) have never been reported for Bromeliaceae. Cryptanthus (Bromelioideae) is chromosomally anomalous within the family at $n=17$, and could, theoretically, correspond to the paleotetraploid level within the model (Fig. 5). If further research were to support Cryptanthus as a true tetraploid (i.e., $8+9$ to 17 ) this would suggest its removal from subfamily Bromelioideae and the establishment of a new subfamily, Cryptanthioideae. However, an alternative, more likely hypothesis must be considered, where Cryptanthus is the product of decreasing aneuploidy from the extant base of $x=25$. Such presumed products of aneuploid reduction are encountered in the genus Tillandsia $(x=$ in T. umbellata André ( $n=18$; Brown et al., $1984)$ and in $T$. complanata $(n=20,22)$ and $T$. leiboldiana $(n=19)$, discussed earlier. Thus the "tetraploid-level" $(n=17)$ in Cryptanthus is very nearly attained in Tillandsia. Additional indirect support for the aneuploid origin of Cryptanthus rests in the fact that the genus exhibits numerous features considered to be derived within the subfamily (e.g., leaves dimorphic, in part; flowers polygamous, in part; stamens adnate to petals; few ovules; pollen 1-lept). Furthermore, aneuploid reduction appears to have occurred in the large Bromelioideae genus Aechmea (175 spp.), and is presumably responsible for the $n=21$ report in A. tillandsioides (Marchant, 1967). At present, we view Cryptanthus as having evolved via aneuploidy.

Intensive study of the reported CryptanthusBillbergia intergeneric hybrids $(C$. beuckeri $\times$ Billbergia nutans and C. bahianus $\times$ Billbergia nutans; Smith and Read, 1975) is needed. These hybrids were generated under horticultural conditions and have received little scientific study beyond the nomenclature work of Smith and Read (1975). Marchant (1967) has reported $n=\mathrm{c} 21$ for $\times$ Cryptbergia meadii (=Cryptanthus beuckeri $\mathrm{E}$. Morren $\times$ Billber- 
gia nutans H. Wendl. ex Regel). A complet $\rightarrow$ Goldblatt, P., AND M. E. Poston. 1988. Observations chromosomal characterization of the parental species and hybrid individuals may provide more definitive clues as to the true chromosomal nature of Cryptanthus (i.e., ancient tetraploid or aneuploid derivative).

\section{LITERATURE CITED}

Billings, F. W. 1904. A study of Tillandsia usneoides. Bot. Gaz. (Crawfordsville) 38: 99-121.

Brown, G. K., AND A. J. Gilmartin. 1983. Chromosomes of bromeliads. J. Bromeliad Soc. 33: 171-172.

$\longrightarrow$, AND - 1984. Stigma structure and variation in Bromeliaceae-neglected taxonomic characters. Brittonia 36: 364-374.

$\longrightarrow$, AND - 1986. Chromosomes of the Bromeliaceae. Selbyana 9: 88-93.

, G. S. Varadarajan, A. J. Gilmartin, and H. LUTHER. 1984. Chromosome number reports LXXXV. Bromeliaceae. Taxon 33: 758-759.

Dahlgren, R., H. T. Clifford, ANd P. F. Yeo. 1985. The families of the monocotyledons: structure, evolution and taxonomy. Springer-Verlag, Berlin.

Di Fulvio, T. E. 1967. Recuentos chromosomicos en angiospermas Argentinas. Kurtziana 4: 87-90.

Frolick, D., AND W. Barthlott. 1987. Micromorphology of epicuticular waxes of monocotyledons. Abstracts, XIV International Botanical Congress, Berlin.

GauTHÉ, J. 1965. Contribution à l'étude caryologiquє des Tillandsiées. Mém. Mus. Natl. Hist. Nat., Sér. B, Bot. 16: 39-59.

Gilmartin, A. J., AND G. K. Brown. 1985. Cleistogamy in Tillandsia capillaris (Bromeliaceae). Biotropica 17: 256-259.

$\longrightarrow$, AND 1 1986a. Bromeliaceae: an international cooperative research project. Taxon 35: 107109.

$\longrightarrow \rightarrow-$ AND $\longrightarrow$. 1986b. Cladistic tests of hypotheses concerning evolution of xerophytes and mesophytes within Tillandsia subgenus Phytarrhiza (Bromeliaceae). Amer. J. Bot. 73: 387-397.

$\longrightarrow \rightarrow-$, AND $\longrightarrow$. 1987. Bromeliales, related monocots, and resolution of relationships among Bromeliaceae subfamilies. Syst. Bot. 12: 493-500. on the chromosome cytology of Velloziaceae. Ann. Missouri Bot. Gard. 75: 192-195.

Grant, V. 1963. The origin of adaptations. Columbia University Press, New York.

- 1981. Plant speciation. Columbia University Press, New York.

LindSChAU, M. 1933. Beiträge zur Zytologie der Bromeliaceae. Planta 20: 506-530.

Marchant. C. J. 1967. Chromosome evolution in the Bromeliaceae. Kew Bull. 21: 161-168.

McWilliams, E. 1974. Chromosome number and evolution. In L. Smith and J. Downs [eds.], Flora neotropica monogr. (Pitcairnioideae) 14: 33-39.

Ranker, T. A., D. E. Soltis, P. S. Soltis, AND A. J. GilmaRTIN. 1988. Molecular systematics of familial and subfamilial relationships of the Bromeliaceae. Amer. J. Bot. 75, part 2: 200.

Sharma, A. K., AND I. GHosh. 1971. Cytotaxonomy of the family Bromeliaceae. Cytologia 36: 237-247.

SMITH, L. B. 1934. Geographic evidence on the lines of evolution in the Bromeliaceae. Bot. Jahrb. Syst. 66: 446-468.

$\rightarrow-$, AND R. J. Downs. 1974. Bromeliaceae (Pitcairnioideae). Flora Neotropica Monogr. 14: 1-658.

$\longrightarrow$, AND -1977 . Bromeliaceae (Tillandsioideae). Flora Neotropica Monogr. 14: 663-1492.

, AND - 1979. Bromeliaceae (Bromelioideae). Flora Neotropica Monogr. 14: 1493-2142.

- AND R. W. READ. 1975. Notes on Bromeliaceae XXXVI. Phytologia 30: 289-303.

Soltis, D. E., A. J. Gilmartin, L. RieseberG, and S. GARDNER. 1987. Genetic variation in the epiphytes Tillandsia ionantha and T. recurvata (Bromeliaceae). Amer. J. Bot. 74: 531-537.

TILL, W. 1984. Sippendifferenzierung Innerhalb Tillandsia subgenus Diaphoranthema in Südamerika mit Besonderer Berücksichtigung des Andenostrandes und der Angrenzenden Gebiete. Ph.D. dissertation, University of Vienna, Austria.

VARADARAJAN, G. S., AND G. K. Brown. 1985. Chromosome number reports LXXXIX. Bromeliaceae. Taxon 34: 729.

Weiss, H. E. 1965. Étude caryologique et cyto-taxonomique de quelques Broméliacées. Mém. Mus. Natl. Hist. Nat., Sér. B, Bot. 16: 9-38. 